\title{
Pseudomonas aeruginosa Virulence Factors Support Voriconazole Effects on Aspergillus fumigatus
}

\author{
Gabriele Sass ${ }^{1,+}$, Pallabi Shrestha ${ }^{1,+}$ (D) and David A. Stevens ${ }^{1,2, *}$ \\ 1 California Institute for Medical Research, San Jose, CA 95128, USA; Gabriele.Sass@cimr.org (G.S.); \\ shrestha.pallabi@gmail.com (P.S.) \\ 2 Division of Infectious Diseases and Geographic Medicine, Department of Medicine, \\ Stanford University School of Medicine, Stanford, CA 94305, USA \\ * Correspondence: stevens@stanford.edu \\ + Both authors contributed equally.
}

check for updates

Citation: Sass, G.; Shrestha, P.; Stevens, D.A. Pseudomonas aeruginosa Virulence Factors Support Voriconazole Effects on Aspergillus fumigatus. Pathogens 2021, 10, 519. https://doi.org/10.3390/ pathogens10050519

Academic Editor: Angela H. Nobbs

Received: 19 March 2021

Accepted: 23 April 2021

Published: 26 April 2021

Publisher's Note: MDPI stays neutral with regard to jurisdictional claims in published maps and institutional affiliations.

Copyright: (c) 2021 by the authors. Licensee MDPI, Basel, Switzerland. This article is an open access article distributed under the terms and conditions of the Creative Commons Attribution (CC BY) license (https:// creativecommons.org/licenses/by/ $4.0 /)$.

\begin{abstract}
Pseudomonas aeruginosa and Aspergillus fumigatus are pathogens that are associated with deterioration of lung function, e.g., in persons with cystic fibrosis (CF). There is evidence that coinfections with these pathogens cause airway inflammation and aggravate pathology in CF lungs. Intermicrobial competition of $P$. aeruginosa and A. fumigatus has been described, but it is unknown how anti-fungal therapy is affected. The anti-fungal azole voriconazole (VCZ), supernatants of P. aeruginosa laboratory isolates PA14 or PAO1, or clinical isolate Pa10 independently inhibited biofilm metabolism of A. fumigatus isolates 10AF and AF13073. When VCZ and supernatants were combined at their $\mathrm{IC}_{50} \mathrm{~s}$, synergistic effects on $A$. fumigatus were found. Synergistic effects were no longer observed when $P$. aeruginosa supernatants were prepared in the presence of iron, or when $P$. aeruginosa mutants were lacking the ability to produce pyoverdine and pyochelin. Combination of pure P. aeruginosa products pyoverdine, pyochelin, and pyocyanin with VCZ showed synergistic anti-fungal effects. Combining VCZ with $P$. aeruginosa supernatants also improved its MIC and MFC against planktonic $A$. fumigatus. In summary, in the case of P. aeruginosa-A. fumigatus coinfections, it appeared that the P. aeruginosa co-infection facilitated therapy of the Aspergillus; lower concentrations of VCZ might be sufficient to control fungal growth.
\end{abstract}

Keywords: Pseudomonas aeruginosa; Aspergillus fumigatus; voriconazole; microbial interaction; cystic fibrosis; drug interaction; therapy

\section{Introduction}

Persons with cystic fibrosis (CF), a hereditary disease caused by mutations in the genes specifying the cystic fibrosis transmembrane conductance regulator (CFTR), a chloride channel on epithelial cells [1-3], frequently suffer from bacterial, fungal, and viral co-morbidities of the lung that aggravate the course of disease. P. aeruginosa, members of the $B$. cepacia complex, A. fumigatus, respiratory syncytial virus, and influenza virus are prominent bacterial, fungal, and viral pathogens impairing lung function, especially when occurring in co-infections [4,5]. Therapies used during CF encompass drugs targeted against CF itself, encompassing two main classes of CFTR-targeting compounds: CFTR potentiators, increasing activity of CFTR on epithelial surfaces, and CFTR correctors, improving defective protein processing and trafficking [6], as well as drugs against each of the microbial infections, or co-infections. Microorganisms, and also drugs, interact, affect, and are affected by individual conditions in the lungs. A. fumigatus infections can include Aspergillus bronchitis, allergic bronchopulmonary aspergillosis (ABPA), and/or chronic progressive aspergillosis, and ultimately contribute to the need for lung transplantation [7]. In anatomically abnormal lungs, Aspergillus would persist as mycelial forms and generate biofilms. First-line treatment of ABPA is performed with oral corticosteroids [8,9], with concomitant side effects. Voriconazole (VCZ), itraconazole, posaconazole, and the anti-IgE 
antibody omalizumab might be alternatives [10-12]. Co-infections with A. fumigatus and $P$. aeruginosa trigger more severe outcomes than each mono-infection $[13,14]$. Interactions of A. fumigatus and $P$. aeruginosa have been studied for many years, with the majority of studies pointing towards an anti-fungal role of $P$. aeruginosa, interfering with fungal metabolism or growth via molecules such as its major siderophore pyoverdine [15], phenazines such as pyocyanin (5-N-methyl-1-hydroxyphenazine) [15-17], 1-hydroxyphenazine [15], phenazine1-carboxamide [15], phenazine-1-carboxylic acid [15], and di-rhamnolipids [16]. We here aimed to illuminate interactions between bacterial molecules and anti-fungal therapy (use of VCZ) affecting A. fumigatus biofilm formation.

\section{Results}

2.1. Determination of $I_{50}$ for P. aeruginosa Strain PA14 Supernatants or VCZ against A. fumigatus $10 A F$ Biofilm Formation

Planktonic supernatants of P. aeruginosa, produced under limiting iron conditions in RPMI medium, contain the major siderophore pyoverdine, which inhibits $A$. fumigatus biofilm metabolism. Here, we determined the $\mathrm{IC}_{50}$ for PA14 supernatants by dilution in RPMI to be between 1:256 and 1:1024 (Figure 1A).
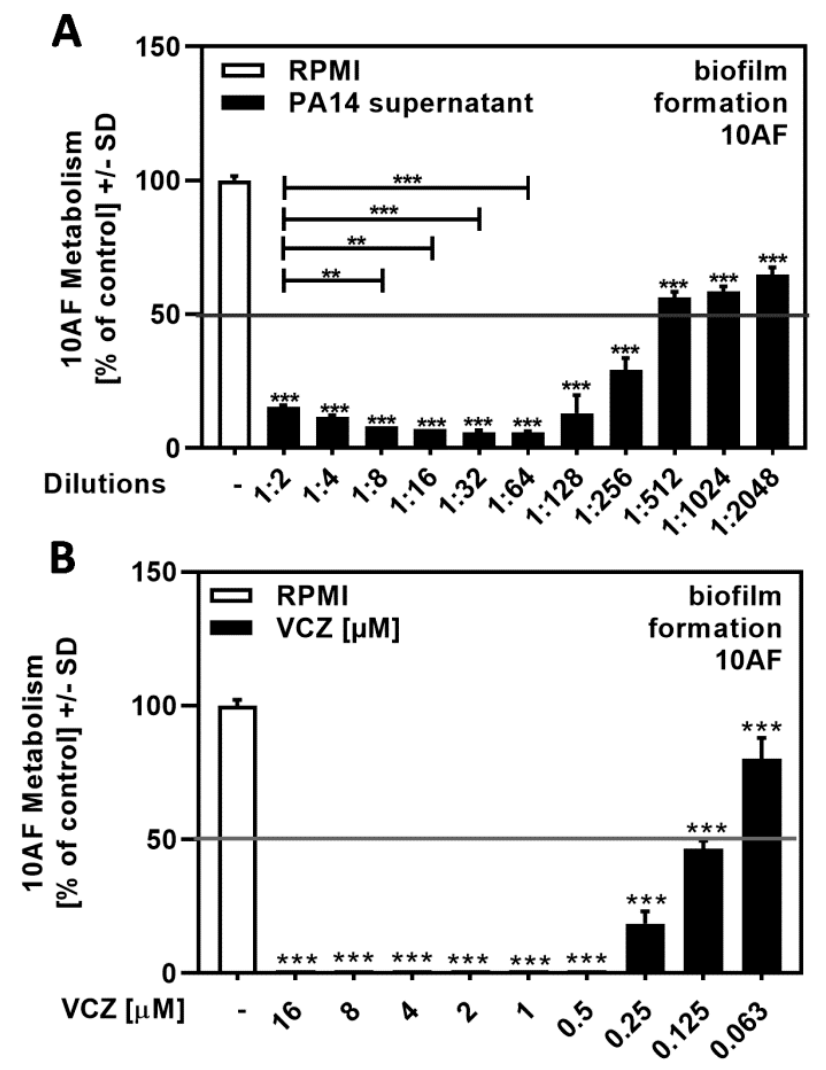

Figure 1. Dose-response curves showing $\mathrm{IC}_{50} \mathrm{~s}$ for the antifungal effects of $P$. aeruginosa wild-type strain PA14 supernatant and VCZ dilutions against 10AF forming biofilm. PA14 wild-type bacteria $\left(5 \times 10^{7} / \mathrm{mL}\right.$ in RPMI 1640 medium) supernatant in twofold serial dilutions (final concentrations: 1:2 to 1:2048) (A) or VCZ in twofold serial dilutions (final concentrations: 16 to $0.063 \mu \mathrm{M}$ ) (B) were added to $10 \mathrm{AF}$ conidia ( $10^{5}$ conidia/mL in RPMI 1640 medium). Assay plates were incubated at $37^{\circ} \mathrm{C}$ overnight. Fungal metabolism was measured by XTT assay. Metabolism in the presence of RPMI alone was regarded as $100 \%$ and compared to each supernatant dilution. Statistical analysis: one-way ANOVA: two asterisks $=p \leq 0.01$, three asterisks $=p \leq 0.001$, respectively. Comparison: RPMI (white bar) vs. all other bars (black bars), or as indicated by the ends of the brackets.

A previous study used VCZ concentrations between 0.125 and $1 \mu \mathrm{M}$ for drug-drug interaction studies, with $A$. fumigatus forming biofilm metabolism as a readout [17]. Here, 
we determined the $\mathrm{IC}_{50}$ for $\mathrm{VCZ}$ against forming biofilm metabolism to be between 0.25 and $0.063 \mu \mathrm{M}$ (Figure 1B).

\subsection{PA14 Supernatant Supported VCZ Anti-Fungal Activity against $10 A F$}

Combining PA14 supernatant at concentrations close to its $\mathrm{IC}_{50}$ (twofold dilutions of 1:256 to 1:1024) with VCZ at concentrations close to its $\mathrm{IC}_{50}(0.25$ to $0.063 \mu \mathrm{M})$ resulted in increased effects on forming 10AF metabolism at all combinations of PA14 supernatants with $0.25 \mu \mathrm{M}$ (Figure 2A) and $0.125 \mu \mathrm{M} \mathrm{VCZ} \mathrm{(Figure} \mathrm{2B).} \mathrm{At} 0.063 \mu \mathrm{M} \mathrm{VCZ} \mathrm{(Figure} \mathrm{2C),}$ only the two lower concentrations of supernatants increased the combined effects. Use of the BLISS Independence Model indicated that the interaction of PA14 supernatants with VCZ was mostly synergistic (Table 1 and Table S1).

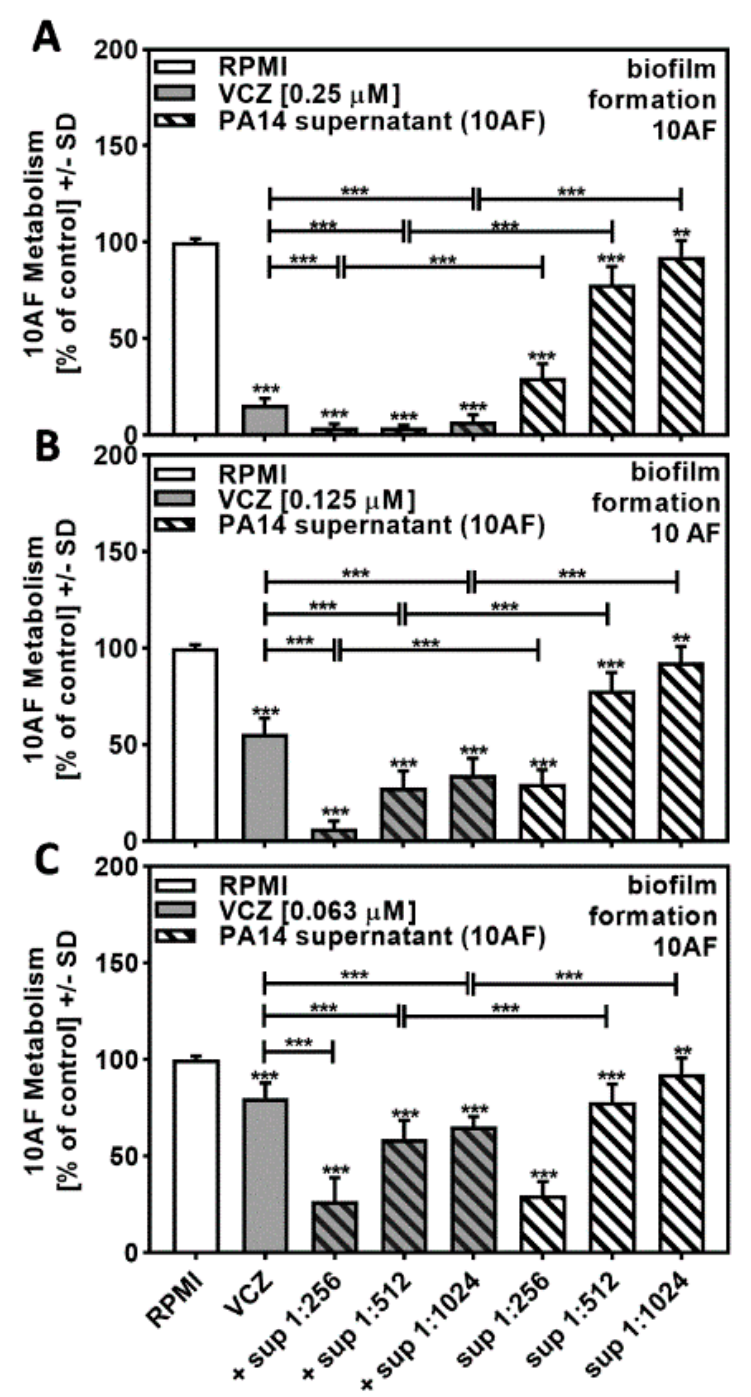

Figure 2. Combination of PA14 supernatant and VCZ significantly increased antifungal effects on forming 10AF biofilm. (A-C) PA14 wild-type bacteria $\left(5 \times 10^{7} / \mathrm{mL}\right.$ in RPMI 1640 medium) supernatant was diluted to final concentrations of 1:256 to 1:1024 and combined with (A) VCZ $0.250 \mu \mathrm{M},(\mathbf{B}) \mathrm{VCZ}$ $0.125 \mu \mathrm{M}$, or $(\mathrm{C}) \mathrm{VCZ} 0.063 \mu \mathrm{M}$ to test their combined antifungal activities against 10AF forming biofilm ( $10^{5}$ conidia/mL in RPMI 1640 medium). Assay plates were incubated at $37^{\circ} \mathrm{C}$ overnight. $10 \mathrm{AF}$ fungal metabolism was measured by XTT assay. Statistical analysis: metabolism in the presence of RPMI alone (white bar) was regarded as $100 \%$, and compared by unpaired $t$-test to VCZ alone (gray bar) and PA14 supernatant dilutions alone (striped bars) and their combinations (gray striped bars). One-way ANOVA: VCZ vs. all VCZ combinations. Unpaired $t$-test for each supernatant dilution vs. its combination with VCZ: two asterisks $=p \leq 0.01$, three asterisks $=p \leq 0.001$. 
Table 1. Bliss Independence Model (PA14 supernatant and VCZ combination effects against 10AF forming biofilm).

\begin{tabular}{cccc}
\hline & VCZ 0.250 & VCZ 0.125 & VCZ 0.063 \\
\hline Pa sup 1:256 & I & S & I \\
\hline Pa sup 1:512 & S & S & S \\
\hline Pa sup 1:1024 & S & S & S \\
\hline
\end{tabular}

(S = synergy, I = independence).

Independent results calculated for the combinations of VCZ 0.250 and $0.063 \mu \mathrm{M}$ with PA14 supernatant 1:256 were a result of strong individual component inhibition of 10AF metabolism. None of the combinations showed antagonistic effects.

\subsection{Synergistic Interaction of VCZ with P. aeruginosa Supernatants Was Independent of the} $P$. aeruginosa or A. fumigatus Strain Used

In order to determine the biological range of VCZ-P. aeruginosa-A. fumigatus interaction, we studied another reference A. fumigatus strain, AF13073, and found similar effects (compare Figure 3A to Figure 2B).

We then studied supernatants of the widely used $P$. aeruginosa reference strain PAO1 and investigated combination of its supernatants with VCZ against 10AF forming biofilm metabolism. Our results showed similar effects as observed for the PA14 supernatant-VCZ interaction (compare Figure 3B to Figure 2B).

The BLISS Independence Model revealed overall synergistic and no antagonistic interactions between VCZ and bacterial supernatants. Table 2A,B was constructed for comparative purposes, whereas the calculation process and comparisons for interactions of bacterial supernatant with additional VCZ concentrations are provided in Table S2.

Table 2. Bliss Independence Model (PA14 supernatant and VCZ combined effect against AF13073 forming biofilm and P. aeruginosa, PAO1 and Pa10, supernatant combined effects with VCZ against 10AF forming biofilm).

\begin{tabular}{|c|c|c|c|}
\hline A & $\begin{array}{l}\text { PA14 Sup } \\
\text { (10AF) }\end{array}$ & $\begin{array}{l}\text { PA14 Sup } \\
\text { (AF13073) }\end{array}$ & $\begin{array}{l}\text { PAO1 Sup } \\
\text { (10AF) }\end{array}$ \\
\hline Pa sup 1:256 & $S$ & I & I \\
\hline Pa sup 1:512 & $S$ & $S$ & $S$ \\
\hline \multirow[t]{2}{*}{ Pa sup 1:1024 } & S & S & S \\
\hline & VCZ 0.125 & VCZ 0.125 & VCZ 0.125 \\
\hline B & $\begin{array}{l}\text { Pa10 Sup } \\
\text { (10AF) }\end{array}$ & & \\
\hline Pa sup 1:256 & I & & \\
\hline Pa sup 1:512 & I & & \\
\hline Pa sup 1:1024 & S & & \\
\hline & VCZ 0.125 & & \\
\hline
\end{tabular}

The most synergistic results were obtained when concentrations of bacterial supernatants were used, which by themselves had weak anti-fungal activity (1:1024 for PA14 and PAO1, 1:256 for Pa10).

Synergy of $P$. aeruginosa supernatants and VCZ against $A$. fumigatus forming biofilms is therefore not restricted to individual bacterial or fungal strains. 
A
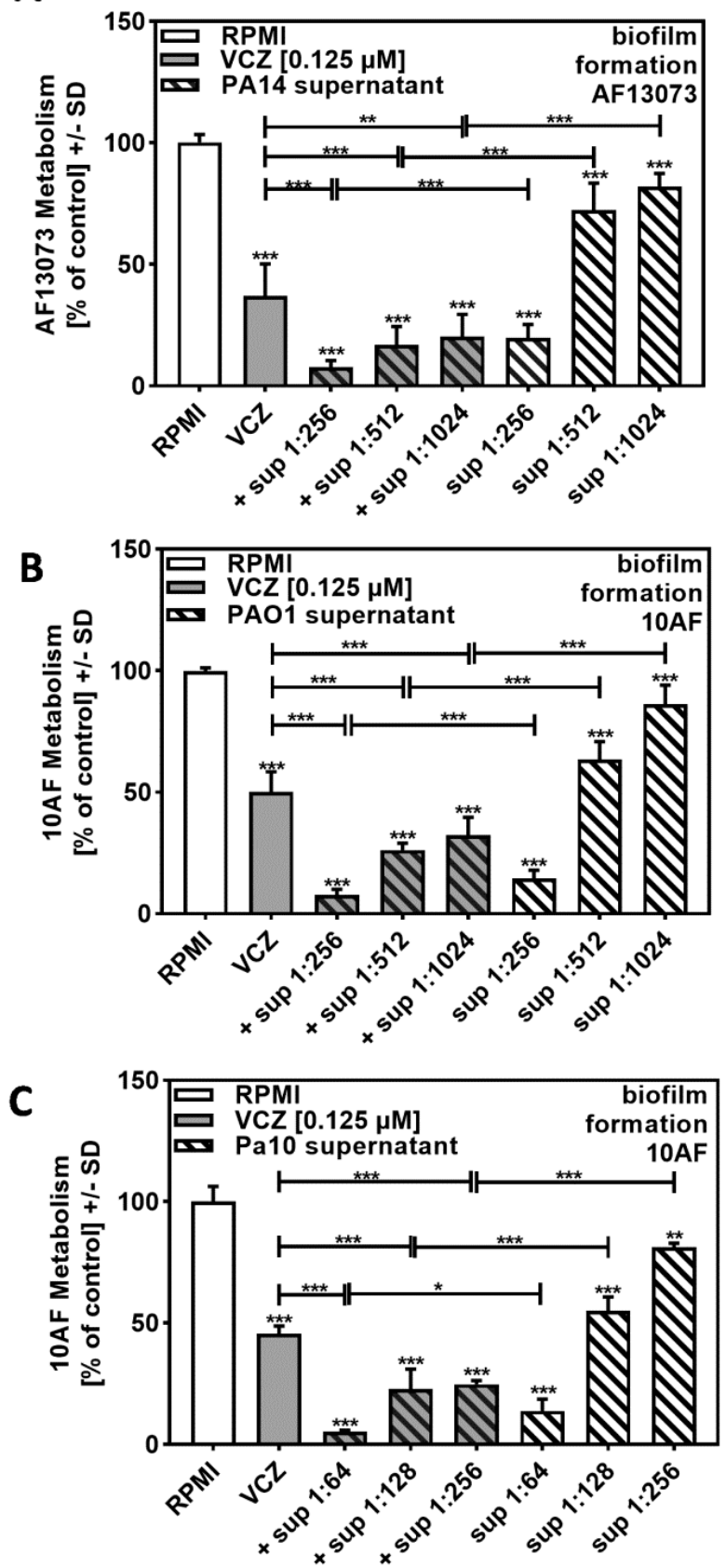

Figure 3. Increased anti-fungal effects by combinations of VCZ and P. aeruginosa supernatants were independent of the bacterial or fungal strains used. PA14 (A), PAO1 (B), or reference non-mucoid CF isolate Pa10 (C) bacteria ( $5 \times 10^{7} / \mathrm{mL}$ in RPMI 1640 medium) supernatants were diluted to final concentrations of 1:256 to 1:1024 (A,B) or 1:64 to 1:256 (C), and combined with VCZ 0.125 $\mu \mathrm{M}$ to test their combined antifungal activities against AF13073 (A) or 10AF-forming biofilm (B,C) ( $10^{5}$ conidia/mL in RPMI 1640 medium). Assay plates were incubated at $37^{\circ} \mathrm{C}$ overnight. Fungal metabolism was measured by XTT assay. Statistical analysis: metabolism in the presence of RPMI alone (white bar) was regarded as $100 \%$ and compared by unpaired $t$-test to VCZ alone (gray bar) and P. aeruginosa supernatant dilutions alone (striped bars) and their combinations (gray striped bars). One-way ANOVA: VCZ vs. all VCZ combinations. Unpaired $t$-test for each supernatant dilution vs. its combination with VCZ: One, two, and three asterisks: $p \leq 0.05, p \leq 0.01, p \leq 0.001$, respectively. 


\subsection{Iron Interfered with P. aeruginosa/VCZ Synergy}

In order to determine molecules in P. aeruginosa supernatants that might contribute to synergistic reactions with $\mathrm{VCZ}$ against $A$. fumigatus forming biofilm metabolism, we prepared PA14 supernatants in the presence of added iron. Iron suppressed the production of the P. aeruginosa major siderophore pyoverdine, thereby decreasing anti-fungal activity. Figure $4 \mathrm{~A}$ shows that bacterial supernatants, prepared in the presence of iron at a dilution of 1:256 or higher, no longer were anti-fungal (compare to Figure 1A) and were not able to act synergistically with VCZ (compare to Figure 2B).
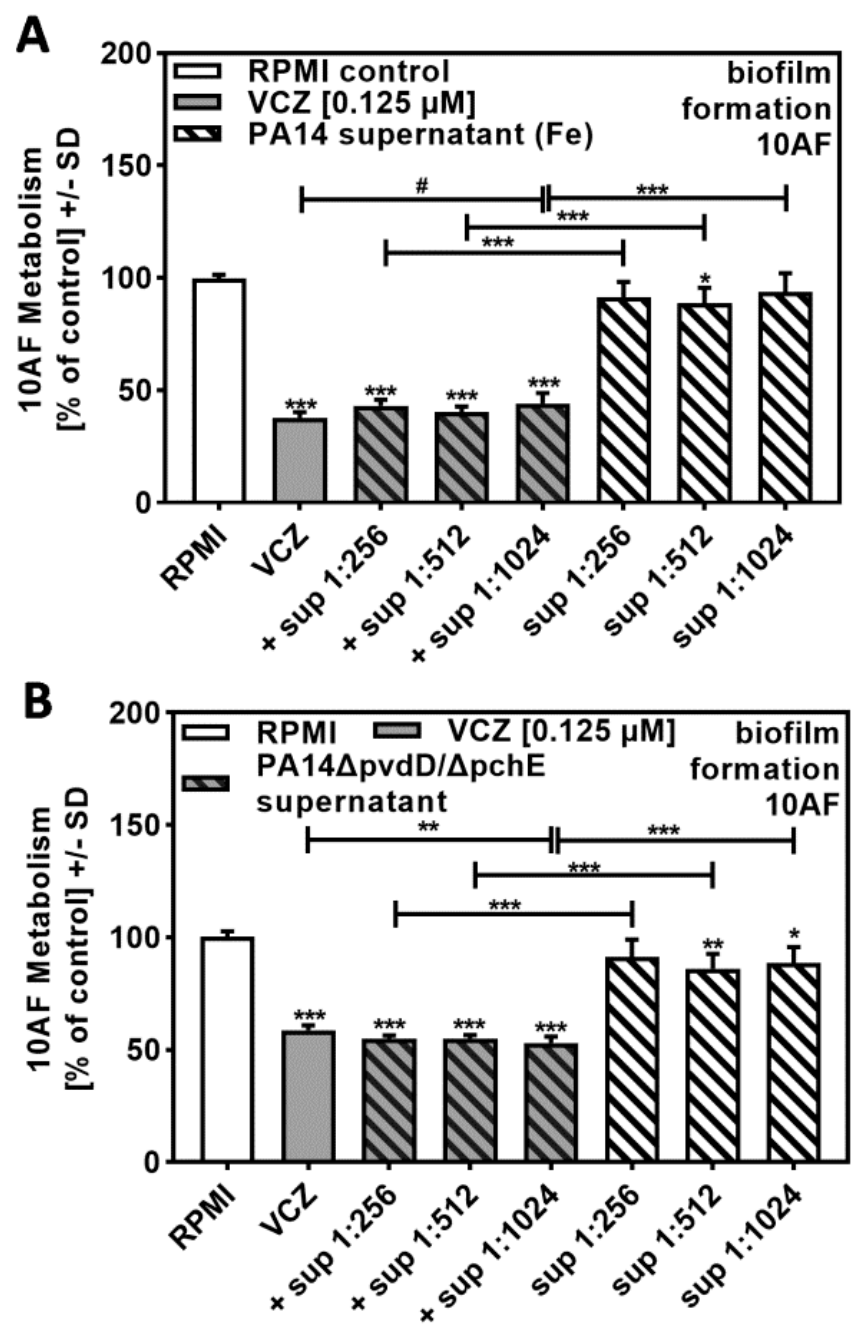

Figure 4. P. aeruginosa siderophores contributed to antifungal effects of VCZ and P. aeruginosa supernatants against $A$. fumigatus forming biofilm metabolism. PA14 supernatants (produced by $5 \times 10^{7}$ bacteria/mL in RPMI 1640 medium, containing $50 \mu \mathrm{M}$ ferric iron) (A), or PA14 $\Delta p v d D / \Delta p c h E$ supernatants (produced by $5 \times 10^{7}$ bacteria/mL in RPMI 1640 medium) (B) were combined with VCZ $(0.125 \mu \mathrm{M})$ at dilutions of 1:256 to 1:1024. 10AF ( $10^{5}$ conidia/mL in RPMI 1640 medium) fungal metabolism was measured by XTT assay. Statistical analysis: metabolism in the presence of RPMI alone (white bar) was regarded as $100 \%$ and compared by unpaired $t$-test to VCZ alone (gray bar) and $P$. aeruginosa supernatant dilutions alone (striped bars) and their combinations (gray striped bars). One-way ANOVA: VCZ vs. all VCZ combinations. Unpaired $t$-test for each supernatant dilution vs. its combination with VCZ: one, two, and three asterisks: $p \leq 0.05, p \leq 0.01, p \leq 0.001$, respectively. Asterisks indicate increased antifungal activities; pound signs indicate decreased antifungal activities. Separate experiments showed that added iron at the concentrations studied did not affect VCZ anti-fungal activity. 
BLISS Independence Model calculation revealed that interactions of supernatants, prepared in the presence of added iron and VCZ, were mostly antagonistic. Table 3, for comparative reasons using a VCZ concentration of $0.125 \mu \mathrm{M}$, showed antagonistic results throughout, whereas combinations of bacterial supernatants with additional VCZ concentrations also partially showed independent results (Table S3).

Table 3. Bliss Independence Model (PA14 supernatant in RPMI with iron, and PA14 $\Delta p v d D / \Delta p c h E$ supernatant combination effects with VCZ against 10AF forming biofilm).

\begin{tabular}{|c|c|c|}
\hline & $\begin{array}{c}\text { PA14 Sup } \\
\text { (In RPMI with Iron) }\end{array}$ & $\operatorname{PA14} \Delta p v d D / \Delta p c h E$ \\
\hline Pa sup 1:256 & $\mathrm{A}$ & I \\
\hline Pa sup 1:512 & $\mathrm{A}$ & $\mathrm{A}$ \\
\hline \multirow[t]{2}{*}{ Pa sup 1:1024 } & A & I \\
\hline & VCZ 0.125 & VCZ 0.125 \\
\hline
\end{tabular}

Supernatants of a PA14 mutant unable to produce the major siderophores pyoverdine and pyochelin (PA14 $\Delta p v d D / \Delta p c h E$ ) showed some anti-fungal activity of their own (Figure 4B). PA14 $\Delta p v d D / \Delta p c h E$ supernatants in combination with VCZ showed no doseresponse curve, and anti-fungal activities of the combinations were much weaker than observed for wild-type supernatants (compare Figure 4B to Figure 2B).

BLISS Independence Model calculations revealed that interactions were not synergistic, as observed for PA14 wild-type (compare Table 3 to Table 1). Further BLISS Independence Model calculations and interactions of bacterial supernatant with additional VCZ concentrations are provided in Table S3.

These data suggest that either pyoverdine or pyochelin, or a combination of both, could explain the positive interaction of $P$. aeuginosa supernatants with VCZ against $A$. $f u$ migatus forming biofilm metabolism.

\subsection{Pyoverdine Contributed to P. aeruginosa/VCZ Synergy}

A dose-response study with pure pyoverdine indicated $\mathrm{its}_{\mathrm{IC}} \mathrm{IC}_{50}$ to be approximately 0.32 to $0.16 \mu \mathrm{M}$ (Figure 5A). Combining pure pyoverdine with VCZ revealed synergy against forming 10AF biofilm metabolism (Figure 5B, Table S4). Pyoverdine therefore enhanced VCZ anti-fungal activity.

\subsection{Pyochelin Contributed to P. aeruginosa/VCZ Synergy}

Performing the same experiments as described in Figure 5, this time using pure pyochelin, we found no or weak anti-fungal activity for concentrations up to $100 \mu \mathrm{M}$ (Figure 6A) but synergy of 25 to $100 \mu \mathrm{M}$ pyochelin with VCZ (Figure 6B, Table S5). It is curious that lower concentrations of pyochelin in $6 \mathrm{~B}$ had stronger effects in combination with VCZ compared to higher concentrations.

\subsection{Pyocyanin Contributed to P. aeruginosa/VCZ Synergy}

Figure $4 \mathrm{~A}$ revealed that high dilutions of iron-rich Pa supernatants (1:256 to 1:1024) did not act synergistically with VCZ, and Figures $5 \mathrm{~B}$ and $6 \mathrm{~B}$ confirm the contribution of pyoverdine and pyochelin, respectively. Under non-limiting iron conditions, Pa not only represses pyoverdine production but also increases production of pyocyanin and other phenazines, which then exert anti-fungal activity. As iron-induced molecules might be diluted to ineffective concentrations when using 1:256 and higher dilutions of bacterial supernatants, we repeated the experiment shown in Figure 4A that involved high dilutions of iron-rich Pa supernatants combined with VCZ by using lower dilutions of PA14 supernatants that contain higher amounts of pyocyanin/phenazines. Our results showed decreased anti-fungal activity of iron-rich supernatants at dilutions of 1:4 to 1:8 
in comparison to iron-limited supernatants (compare Figure 7A to Figure 1A), as well as increased anti-fungal activity in combination with VCZ (Figure 7A, Table S6).
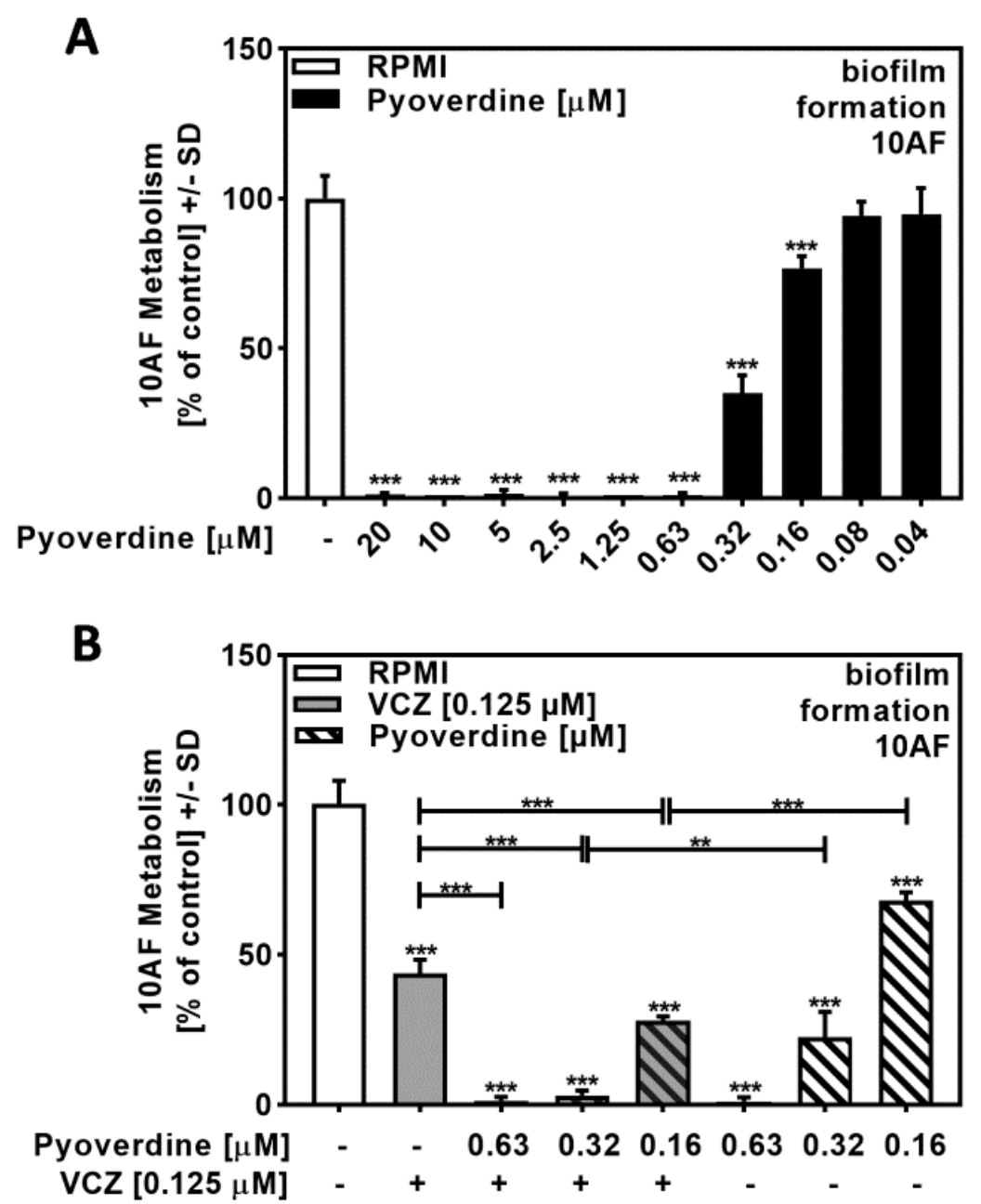

Figure 5. Pyoverdine significantly increased antifungal effects of VCZ against $A$. fumigatus forming biofilm metabolism. (A) Dilutions of pure pyoverdine in RPMI (20 to $0.04 \mu \mathrm{M})$ were tested for activity against forming 10AF ( $10^{5}$ conidia/mL in RPMI 1640 medium) biofilm metabolism. RPMI alone (white bar) was regarded as $100 \%$ and was compared to pyoverdine dilutions (black bars). Statistical analysis: one-way ANOVA: three asterisks: $p \leq 0.001$. (B) Pure pyoverdine $(0.063,0.032$, and $0.016 \mu \mathrm{M})$ was combined with VCZ $(0.125 \mu \mathrm{M})$ and tested for combined antifungal activity against 10AF ( $10^{5}$ conidia/mL in RPMI 1640 medium) forming biofilm. 10AF fungal metabolism was measured by XTT assay. Statistical analysis: metabolism in the presence of RPMI alone (white bar) was regarded as $100 \%$ and compared by unpaired $t$-test to VCZ alone (gray bar), pyoverdine concentrations alone (striped bars), and their combinations (gray striped bars). One-way ANOVA: VCZ vs. all VCZ combinations. Unpaired $t$-test for each pyoverdine concentration vs. its combination with VCZ: two or three asterisks: $p \leq 0.01, p \leq 0.001$, respectively. 

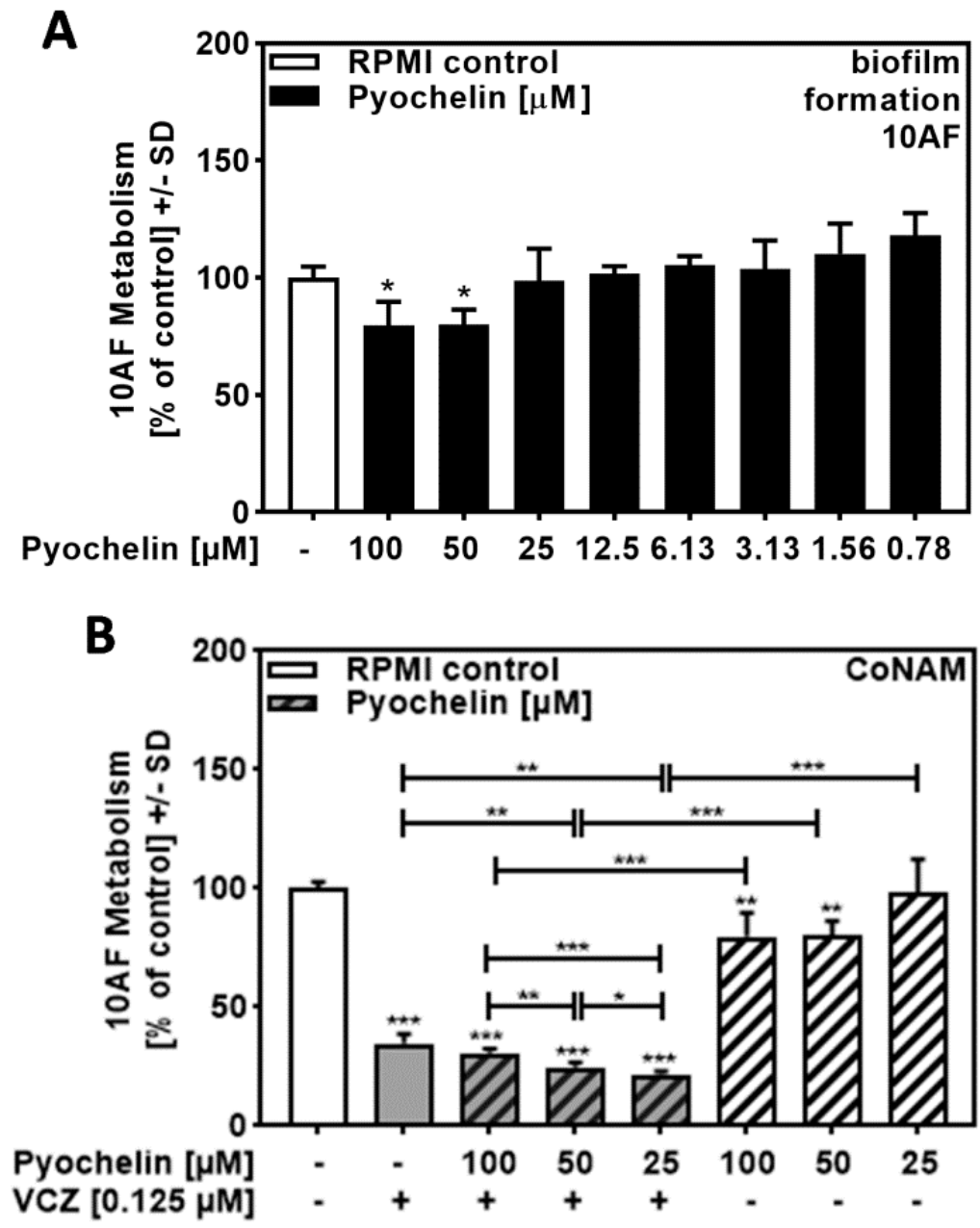

Figure 6. Pyochelin significantly increased anti-fungal effects of VCZ against A. fumigatus forming biofilm metabolism. (A) Dilutions of pure pyochelin in RPMI (100 to $0.78 \mu \mathrm{M}$ ) were tested for anti-fungal activity using forming 10AF ( $10^{5}$ conidia/mL in RPMI 1640 medium) biofilm assays. RPMI alone (white bar) was regarded as $100 \%$ and compared to all pyoverdine dilutions (black bars). Statistical analysis: one-way ANOVA: one asterisk: $p \leq 0.05$. (B) Pure pyochelin (100, 50, and $25 \mu \mathrm{M})$ was combined with VCZ $(0.125 \mu \mathrm{M})$ to test for $10 \mathrm{AF}\left(10^{5}\right.$ conidia/mL in RPMI 1640 medium $)$ forming biofilm metabolism. 10AF metabolism was measured by XTT assay. Statistical analysis: metabolism in the presence of RPMI alone (white bar) was regarded as $100 \%$ and compared by unpaired $t$-test to $\mathrm{VCZ}$ alone (gray bar), pyochelin concentrations alone (striped bars), and their combinations (gray striped bars). One-way ANOVA: VCZ vs. all VCZ combinations. Unpaired $t$-test for each pyochelin concentration vs. its combination with VCZ: one, two, and three asterisks: $p \leq 0.05, p \leq 0.01, p \leq 0.001$, respectively.

As these supernatants do not contain pyoverdine (as verified by missing blue fluorescence under UV light [18]), but contain anti-fungal phenazines, we then tested for combined anti-fungal effects of pure pyocyanin, a phenazine induced by iron, and VCZ, and found positive interaction (Figure 7B, Table S6).

In summary, VCZ anti-fungal activity is supported by pyoverdine, pyochelin, and pyocyanin, depending on the milieu being limited, or not limited, for iron. 


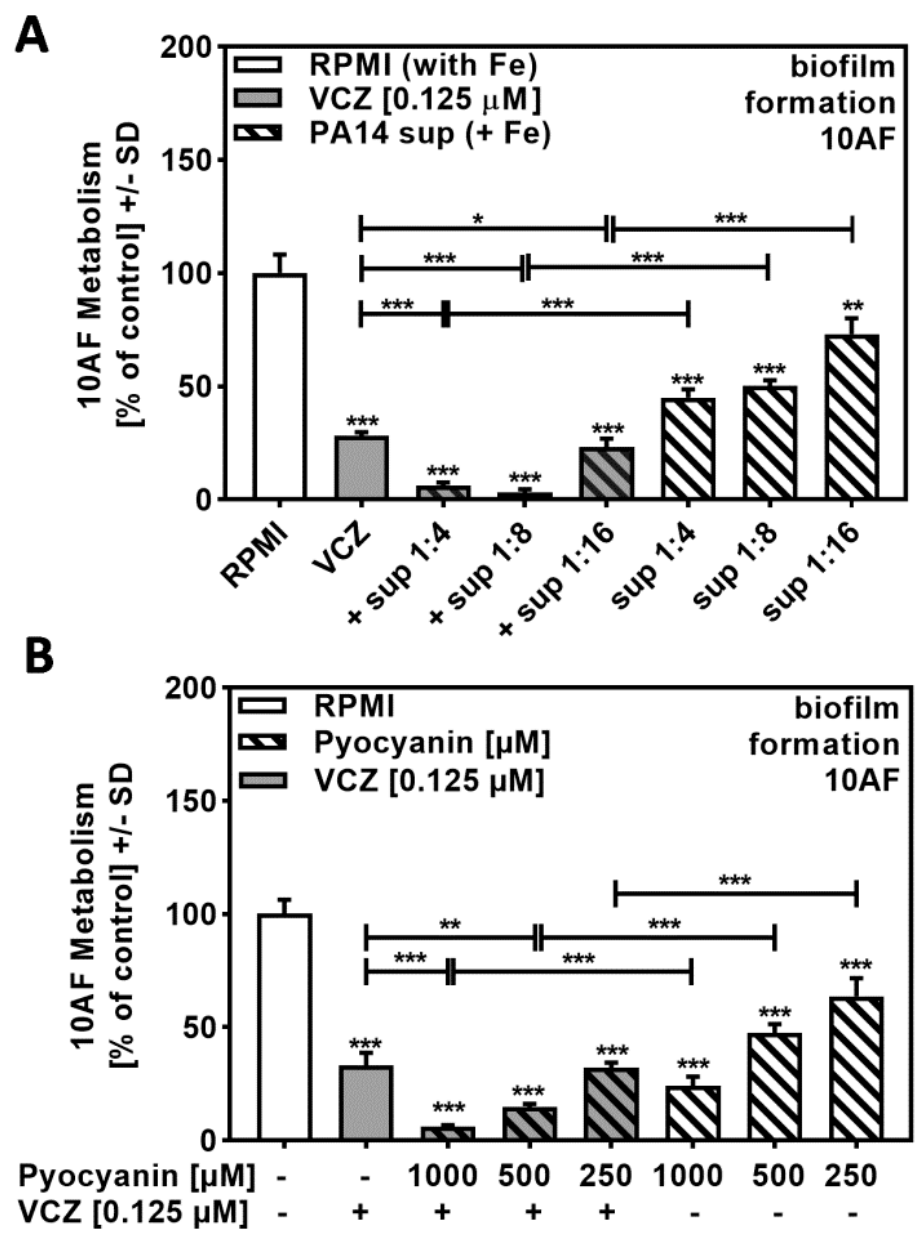

Figure 7. Phenazines/pyocyanin significantly increased anti-fungal effects of VCZ against A. fumigatus forming biofilm metabolism. (A) PA14 supernatant (produced by $5 \times 10^{7}$ bacteria/mL in RPMI 1640 medium, containing $50 \mu \mathrm{M}$ ferric iron, $\left.\mathrm{FeCl}_{3}\right)$ was combined with $\mathrm{VCZ}(0.125 \mu \mathrm{M})$ at dilutions of 1:4 to 1: 16. 10AF ( $10^{5}$ conidia/mL in RPMI 1640 medium) fungal metabolism was measured by XTT assay. Statistical analysis: metabolism in the presence of RPMI alone (white bar) was regarded as $100 \%$ and compared by unpaired $t$-test to VCZ alone (gray bar) and P. aeruginosa supernatant dilutions alone (striped bars) and their combinations (gray striped bars). One-way ANOVA: VCZ vs. all VCZ combinations. Unpaired $t$-test for each supernatant dilution vs. its combination with VCZ: One, two, or three asterisks: $p \leq 0.05, p \leq 0.01, p \leq 0.001$, respectively. Separate experiments showed that added iron at the concentrations studied did not affect VCZ anti-fungal activity. (B) Pure pyocyanin $(1000,500$, and $250 \mu \mathrm{M})$ was combined with VCZ $(0.125 \mu \mathrm{M})$ to test their combined anti-fungal effects against 10AF ( $10^{5}$ conidia/mL in RPMI 1640 medium) forming biofilm metabolism. 10AF fungal metabolism was measured by XTT assay. Statistical analysis: metabolism in the presence of RPMI alone (white bar) was regarded as $100 \%$ and compared by unpaired $t$-test to VCZ alone (gray bar), pyocyanin concentrations alone (striped bars), and their combinations (gray striped bars). One-way ANOVA: VCZ vs. all VCZ combinations. Unpaired $t$-test for each pyocyanin concentration vs. its combination with VCZ: two or three asterisks: $p \leq 0.01, p \leq 0.001$, respectively.

2.8. PA14 Supernatant Supported Anti-Fungal Activity of VCZ against Planktonic A. fumigatus Growth

We tested combined anti-fungal effects against planktonic $A$. fumigatus growth. We found that the combination with $P$. aeruginosa supernatants improved the MIC and MFC of VCZ with strong synergistic effects (Table 4). This corroborates the findings with A. fumigatus biofilms. 
Table 4. MIC, MFC, FICi, and FFCi values for PA14 supernatants, VCZ, as well as combinations of both against planktonic 10AF growth.

\begin{tabular}{ccccc}
\hline & MIC & MFC & FICi & FFCi \\
\hline PA14 supernatant & $1: 32$ & No MFC & - & - \\
\hline VCZ & $0.5 \mu \mathrm{M}$ & $16 \mu \mathrm{M}$ & - & - \\
\hline Combination & $1: 32+0.125 \mu \mathrm{M}$ & $1: 256+8 \mu \mathrm{M}$ & 0.28 & 0.5 \\
\hline
\end{tabular}

\section{Discussion}

A. fumigatus-P. aeruginosa coinfections trigger more severe outcomes than each monoinfection $[13,14]$. This could be a result of inflammatory signals caused by intermicrobial competition. Several $P$. aeruginosa molecules have been identified that interfere with fungal metabolism or growth via molecules such as phenazines, e.g., pyocyanin, or dirhamnolipids [16]. Using defined media and an array of $P$. aeruginosa mutants, we recently found that under low iron conditions, pyoverdine is the major anti-fungal P. aeruginosa product, inhibiting A. fumigatus metabolism and growth by binding and withholding ferric iron from the fungus. Under high iron conditions, P. aeruginosa no longer produces pyoverdine, and thus anti-fungal effects of phenazines become more prominent [19]. Lungs of persons with $\mathrm{CF}$ tend to have higher iron content than infected non-CF lungs or healthy lungs [20]. The iron content in infected lungs varies by compartments, with mucoid plugs being low in iron [21], whereas micro-hemorrhages and hemoptysis provide higher iron levels. We also could show that the $P$. aeruginosa product 3,4-dihydroxy-2-heptylquinoline (PQS) uniquely affects $A$. fumigatus metabolism in two ways, depending on the concentrations of iron present: inhibiting the fungus under low iron conditions while promoting fungal metabolism under high iron conditions [22].

Treatment of A. fumigatus infections of the lung with azoles is common [10,11]. Given that $P$. aeruginosa products inhibit $A$. fumigatus metabolism, it seemed feasible that some of those products interacted with anti-fungal therapy. In fact, we found synergistic anti-fungal activity between VCZ and P. aeruginosa supernatants that was mediated by e.g., pyoverdine and pyochelin under low iron conditions, and pyocyanin under high iron conditions. VCZ MIC and MFC improved when combined with P. aeruginosa supernatants. There are likely other $P$. aeruginosa products able to synergistically interact with azoles and maybe with other anti-fungal agents that we did not test here. Preliminary results (unpublished) indicate that products of other bacterial species, e.g., the Burkholderia cepacia complex, affect azoles as well.

Through using several P. aeruginosa and A. fumigatus strains, we are confident that synergy between VCZ and Pseudomonas supernatants is a general feature during coinfections. We included a non-mucoid CF isolate (Pa10) and found synergy here as well. We previously could show, in an iron-restricted liquid milieu, that clinical Pa isolates, derived from persons with $\mathrm{CF}$, have stronger anti-fungal activity than isolates derived from non-CF infections; moreover, mucoid $\mathrm{Pa}$ isolates from $\mathrm{CF}$ patients were less antifungal than non-mucoid isolates from CF patients. It remains to be seen if non-mucoid CF isolates have stronger synergistic effects than mucoid CF isolates with azoles.

An interesting phenomenon we observed during our studies was that low-iron P. aeruginosa supernatants at some dilutions increased in anti-fungal activity compared to undiluted or less diluted supernatants (e.g., Figure 1A). This phenomenon could either be explained by an increased protective response by A. fumigatus to high amounts of pyoverdine, or by the presence of pro-fungal factors in bacterial supernatants that at high concentrations mask part of their anti-fungal effects. For its own protection, A. fumigatus releases its siderophores into its growth medium to secure iron and withhold iron from its competitors [23]. Preliminary results show that A. fumigatus strains lacking SidA production also show the phenomenon of $P$. aeruginosa supernatant decreased anti-fungal activity at high concentrations, making it more likely that there also are pro-fungal factors in bacterial supernatants. 
Another interesting phenomenon was observed when the P. aeruginosa product pyochelin was combined with VCZ (Figure 6B). We observed synergistic effects that, contrary to effects observed for other $P$. aeruginosa products, increased with decreasing concentrations. We cannot yet explain this observation, but hypothesize that we might examine pro-fungal effects of pyochelin in this situation.

In bacterial co-infections, VCZ might be effective at lower doses compared to $A$. fumigatus mono-infections. This might allow for the use of lower doses of VCZ and help to avoid azole side effects. The presence of certain coinfections, or the treatment for some mono-infections, will alter the subsequent microbiome [24]; can alter the subsequent clinical course; and could make initiation of, and choice of, antifungal therapy more cogent. Studies of any interactions with antifungal therapy need increased attention in view of some reports indicating rising antifungal resistance in Af isolates [25-29].

Although bacterial virulence factors would be unlikely to be clinically useful as antifungal agents, we see a wide field for the discovery of new agents here but would like to caution the community to the fact that treating bacterial co-infections of aspergillosis might require an adjustment in the dose of the anti-fungal used for therapy, and that treatment of Pseudomonas might make therapy of A. fumigatus more difficult.

\section{Materials and Methods}

\subsection{Materials}

Pyocyanin, pyoverdine, pyochelin, ferric iron $\left(\mathrm{FeCl}_{3}\right)$, 2,3-bis(2-methoxy-4-nitro-5sulfophenyl)-2H-tetrazolium-5-carboxanilide inner salt (XTT), menadione, and RPMI 1640 medium were purchased from Sigma-Aldrich (St. Louis, MO, USA). Iron contents in RPMI 1640 medium were below the detection limit $(<1 \mu \mathrm{M}$, measured by inductively coupled plasma optical emission spectroscopy; Paolo Visca, Rome, Italy, personal communication). Voriconazole was obtained from Pfizer, New York City. Stock was prepared in DMSO and was further diluted to test conditions in RPMI. DMSO concentration in our combination experiments was $0.01 \%$. DMSO concentrations below $1 \%$ do not affect $A$. fumigatus biofilm metabolism, and thus did not require dedicated DMSO controls. Large batches of the reagents were prepared in aliquots and frozen, and a fresh aliquot was used in each experiment.

\subsection{Strains and Isolates}

All bacterial and fungal strains used in this study are provided in Table 5. The use of all microbes in our laboratory is approved by the CIMR Biological Use Committee (approval No. 001-03Yr.15).

Table 5. Strains and isolates used in this study.

\begin{tabular}{|c|c|c|c|c|}
\hline Organism & Isolate & Description & ATCC & References \\
\hline $\begin{array}{l}\text { Aspergillus } \\
\text { fumigatus }\end{array}$ & $10 \mathrm{AF}$ & Virulent patient isolate & 90,240 & {$[30,31]$} \\
\hline $\begin{array}{l}\text { Aspergillus } \\
\text { fumigatus }\end{array}$ & AF13073 & & 13,073 & \\
\hline $\begin{array}{l}\text { Pseudomonas } \\
\text { aeruginosa }\end{array}$ & PA14 & $\begin{array}{c}\text { Parental strain of all PA14 } \\
\text { mutants studied }\end{array}$ & & [32-34] \\
\hline $\begin{array}{l}\text { Pseudomonas } \\
\text { aeruginosa }\end{array}$ & PAO1 & & 15,692 & {$[35]$} \\
\hline $\begin{array}{c}\text { Pseudomonas } \\
\text { aeruginosa }\end{array}$ & Pa10 & $\begin{array}{l}\text { Reference non-mucoid CF } \\
\text { isolate }\end{array}$ & & [36] \\
\hline $\begin{array}{c}\text { Pseudomonas } \\
\text { aeruginosa }\end{array}$ & $\mathrm{PA} 14 \Delta p v d D / \Delta p c h E$ & $\begin{array}{c}\text { Pyoverdine-pyochelin } \\
\text { double siderophore } \\
\text { mutant }\end{array}$ & & \\
\hline
\end{tabular}




\subsection{P. aeruginosa Planktonic Supernatant Production and Dilution}

P. aeruginosa supernatants were prepared as detailed previously [36]. Briefly, P. aeruginosa wild-type or mutant bacteria $\left(5 \times 10^{7}\right.$ cells $\left./ \mathrm{mL}\right)$ were incubated in RPMI 1640 medium (Sigma-Aldrich) with or without the addition of $50 \mu \mathrm{M} \mathrm{FeCl}_{3}$ at $37^{\circ} \mathrm{C}$ and $100 \mathrm{rpm}$ for $24 \mathrm{~h}$. Bacterial cultures were centrifuged at $2000 \mathrm{rpm}$ for $30 \mathrm{~min}$ at room temperature and filtered for sterility $(0.22 \mu \mathrm{m})$. Supernatants were diluted in RPMI in 1:2 steps with final concentrations ranging from 1:2 to 1:2048.

\subsection{Assay for Measurement of Aspergillus Forming Biofilm Metabolism}

A. fumigatus conidia $\left(10^{5} / \mathrm{mL}\right.$ final concentration) were distributed into the wells of sterile flat-bottom 96-well culture plates at $50 \mu \mathrm{L} /$ well. Bacterial supernatants or test substances and VCZ were combined in equal parts by volume ( $25 \mu \mathrm{L}$ each) to the final concentrations indicated. Final volumes in wells during assays were $100 \mu \mathrm{L}$. RPMI 1640 medium served as the negative control. The assay plates were incubated at $37^{\circ} \mathrm{C}$ overnight, and hyphae growth was verified by optical microscopy before performing XTT assays.

All assays were evaluated by XTT metabolic assay as detailed previously $[36,37]$. Briefly, $150 \mu \mathrm{L}$ of an XTT/menadione mixture ( $150 \mu \mathrm{g} / \mathrm{mL}$ XTT, $30 \mu \mathrm{M}$ menadione) were added to each test well and incubated at $37{ }^{\circ} \mathrm{C}$ for $1 \mathrm{~h}$. Supernatants from each well were transferred to a fresh 96 well plate $(100 \mu \mathrm{L})$ and assayed using a plate reader (Vmax, Molecular Devices, San Jose, CA, USA) at $490 \mathrm{~nm}$.

\subsection{Minimal Inhibitory Concentration (MIC), Minimal Fungicidal Concentration (MFC), Fractional Inhibitory, and Fungicidal Concentration Indexes (FICi and FFCi)}

Fifty microliters of VCZ (drug A, range $0.09-45.8 \mu \mathrm{M}$, corresponding to $0.032-16 \mu \mathrm{g} / \mathrm{mL}$ ) was distributed in rows and $50 \mu \mathrm{L}$ of $P$. aeruginosa supernatant (drug B, range 1:10-1:2560) was distributed in the columns for the interaction. Nine hundred microliters of standardized inoculum were added to the tubes. Tubes were incubated for $48 \mathrm{~h}$ at $35^{\circ} \mathrm{C}$ before reading of MICs. Fractional inhibitory concentration index (FICi) was determined by the equation: $\mathrm{FICi}=\left(\mathrm{MIC}_{\mathrm{A}}\right.$ in combination $/ \mathrm{MIC}_{\mathrm{A}}$ tested alone $)+\left(\mathrm{MIC}_{\mathrm{B}}\right.$ in combination $/ \mathrm{MIC}_{\mathrm{B}}$ tested alone). The fractional fungicidal concentration index (FFCi) was calculated and interpreted in the same way as described for FICi. The assay was performed in duplicate.

Drug interactions were classified as strong synergism when FICi or FFCi $<0.5$; weak synergism when $0.5 \leq$ FICi or FFCi $<1$; additive when $1 \leq$ FICi or FFCi $<2$; indifferent when FICi or FFCi $=2$; and antagonistic when FICi or FFCi $>2$ [38].

To determine the minimum fungicidal concentration (MFC), we plated $50 \mu \mathrm{L}$ of each tube without visual growth on Sabouraud agar and incubated the mixture at $35^{\circ} \mathrm{C}$ for $24 \mathrm{~h}$. MFC was considered the minimal concentration of the drug resulting in killing $\geq 99 \%$ of the inoculum.

\subsection{BLISS Independence Model for Analysis of Drug Combination Effects}

Combined drug effects were also calculated using the BLISS Independence Model as described previously [39]. Briefly, if drugs A (VCZ) and B (P. aeruginosa supernatant) inhibit $Y_{a}$ and $Y_{b}$ percent of growth, respectively, their predicted combined effect (considering they work independently) is given by the following formula: $\mathrm{YP}_{a b}=Y_{a}+Y_{b}-Y_{a} Y_{b}$. The predicted combined effect is compared to the observed combined effect (anti-fungal activity by the drug combination in XTT assays). The result is interpreted as

- $\quad$ Observed > Predicted: Synergy

- $\quad$ Observed $=$ Predicted: Independent $\left(5 \%\right.$ range of $\left.\mathrm{YP}_{\mathrm{ab}}\right)$

- $\quad$ Observed < Predicted: Antagonism

(abbreviations: $\mathrm{S}=$ synergy, $\mathrm{I}=$ independence, $\mathrm{A}=$ antagonism, $\mathrm{Y}_{\mathrm{a}}=$ inhibition of fungal metabolism by respective $\mathrm{VCZ}, \mathrm{Y}_{\mathrm{b}}=$ inhibition of fungal metabolism by respective Pa sup, $\mathrm{Y}_{\mathrm{ab}}^{\mathrm{o}}=$ observed combined antifungal effect, $\mathrm{YP}_{\mathrm{ab}}=$ predicted combined antifungal effect). 


\subsection{Statistical Analysis}

Results were analyzed using Student's $t$-test if two groups were compared, and oneway ANOVA combined with a Tukey's post-test for multiple comparisons. All data in this study are expressed as a mean $\pm \mathrm{SD}$. Data reported as the percent of control were compared using Student's $t$-test after arcsin transformation of the proportions; these data are presented as the percentages. Each assay was performed with three to eight biological and technical replicates. Representative experiments are shown.

Supplementary Materials: The following are available online at https:/ /www.mdpi.com/article/10 .3390/pathogens10050519/s1, Table S1: Bliss Independence Model calculation: PA14 supernatant and VCZ $(\mu \mathrm{M})$ combined effects against 10AF forming biofilm. Table S2: Bliss Independence Model calculation: PA14 supernatant and VCZ $(\mu \mathrm{M})$ combined effects against AF13073 forming biofilm and P. aeruginosa, PAO1 and Pa10, supernatants, combined with VCZ against 10AF forming biofilm. Table S3: Bliss Independence Model calculation: PA14 supernatant (in RPMI with iron, concentrations 1:256 to 1:1024) or PA14 $\Delta \mathrm{pvdD} / \Delta \mathrm{pchE}$ supernatant at low concentrations (1:256 to 1:1024), combined with VCZ $(\mu \mathrm{M})$; combined effects against 10AF forming biofilm. Table S4: Bliss Independence Model calculation: pyoverdine $(\mu \mathrm{M})$ and VCZ $(\mu \mathrm{M})$ combination effects against $10 \mathrm{AF}$ forming biofilm. Table S5: Bliss Independence Model calculation: pyochelin $(\mu \mathrm{M})$ and VCZ $(\mu \mathrm{M})$ combination effects against 10AF forming biofilm. Table S6: Bliss Independence Model calculation: upper part: PA14 supernatant (in RPMI with iron concentrations 1:4 to 1:16), lower part: pyocyanin $(\mu \mathrm{M})$. Combination effects with VCZ $(\mu \mathrm{M})$ against 10AF forming biofilm.

Author Contributions: Conceptualization, D.A.S.; data curation, D.A.S., G.S., and P.S.; formal analysis, D.A.S., G.S., and P.S.; funding acquisition, D.A.S.; investigation, D.A.S., G.S., and P.S.; methodology, D.A.S., G.S., and P.S.; project administration, D.A.S. and G.S.; resources, D.A.S.; software, G.S.; supervision, D.A.S. and G.S.; validation, D.A.S., G.S., and P.S.; visualization, D.A.S., G.S., and P.S.; writing — original draft, G.S. and P.S.; writing-review and editing, D.A.S. and G.S. All authors have read and agreed to the published version of the manuscript.

Funding: This research was funded by the Foundation for Research in Infectious Diseases (FRID, grant 8201), grant number 8201.

Institutional Review Board Statement: Not applicable.

Informed Consent Statement: Not applicable.

Data Availability Statement: Raw data for these studies are available from Gabriele.Sass@cimr.org.

Acknowledgments: The authors thank Eric Déziel (INRS-Armand-Frappier Santé Biotechnologie, Laval, Québec, Canada) for providing the PA14 $\Delta p v d D / \Delta p c h E$ mutant, and Marife Martinez for excellent technical support.

Conflicts of Interest: The authors declare no conflict of interest. The funders had no role in the design of the study; in the collection, analyses, or interpretation of data; in the writing of the manuscript; or in the decision to publish the results.

\section{References}

1. Riordan, J.R.; Rommens, J.M.; Kerem, B.; Alon, N.; Rozmahel, R.; Grzelczak, Z.; Zielenski, J.; Lok, S.; Plavsic, N.; Chou, J.L.; et al. Identification of the cystic fibrosis gene: Cloning and characterization of complementary DNA. Science 1989, 245, $1066-1073$. [CrossRef]

2. Collins, F.; Jurivich, D.; Sistonen, L.; Kroes, R.; Morimoto, R. Cystic fibrosis: Molecular biology and therapeutic implications. Science 1992, 256, 774-779. [CrossRef]

3. Rowe, S.M.; Miller, S.; Sorscher, E.J. Cystic fibrosis. N. Engl. J. Med. 2005, 352, 1992-2001. [CrossRef]

4. King, J.; Brunel, S.F.; Warris, A. Aspergillus infections in cystic fibrosis. J. Infect. 2016, 72, S50-S55. [CrossRef]

5. O'Brien, S.; Fothergill, J.L. The role of multispecies social interactions in shaping Pseudomonas aeruginosa pathogenicity in the cystic fibrosis lung. FEMS Microbiol. Lett. 2017, 364, fnx128. [CrossRef]

6. Gentzsch, M.; Mall, M.A. Ion Channel Modulators in Cystic Fibrosis. Chest 2018, 154, 383-393. [CrossRef]

7. Baxter, C.G.; Dunn, G.; Jones, A.M.; Webb, K.; Gore, R.; Richardson, M.D.; Denning, D.W. Novel immunologic classification of aspergillosis in adult cystic fibrosis. J. Allergy Clin. Immunol. 2013, 132, 560-566. [CrossRef] [PubMed]

8. Moss, R.B. Fungi in Cystic Fibrosis and Non-Cystic Fibrosis Bronchiectasis. Semin. Respir. Crit. Care Med. 2015, 36, 207-216. [CrossRef] 
9. Agarwal, R.; Chakrabarti, A.; Shah, A.; Gupta, D.; Meis, J.F.; Guleria, R.; Moss, R.; Denning, D.W.; ABPA complicating asthma ISHAM working group. Allergic bronchopulmonary aspergillosis: Review of literature and proposal of new diagnostic and classification criteria. Clin. Exp. Allergy 2013,43, 850-873. [CrossRef]

10. Chishimba, L.; Niven, R.M.; Cooley, J.; Denning, D.W. Voriconazole and Posaconazole Improve Asthma Severity in Allergic Bronchopulmonary Aspergillosis and Severe Asthma with Fungal Sensitization. J. Asthma 2012, 49, 423-433. [CrossRef]

11. Hogan, C.; Denning, D.W. Allergic Bronchopulmonary Aspergillosis and Related Allergic Syndromes. Semin. Respir. Crit. Care Med. 2011, 32, 682-692. [CrossRef]

12. Perisson, C.; Destruys, L.; Grenet, D.; Bassinet, L.; Derelle, J.; Sermet-Gaudelus, I.; Thumerelle, C.; Prevotat, A.; Rosner, V.; Clement, A.; et al. Omalizumab treatment for allergic bronchopulmonary aspergillosis in young patients with cystic fibrosis. Respir. Med. 2017, 133, 12-15. [CrossRef]

13. Amin, R.; Dupuis, A.; Aaron, S.D.; Ratjen, F. The Effect of Chronic Infection with Aspergillus fumigatus on Lung Function and Hospitalization in Patients with Cystic Fibrosis. Chest 2010, 137, 171-176. [CrossRef] [PubMed]

14. Reece, E.; Segurado, R.; Jackson, A.; McClean, S.; Renwick, J.; Greally, P. Co-colonisation with Aspergillus fumigatus and Pseudomonas aeruginosa is associated with poorer health in cystic fibrosis patients: An Irish registry analysis. BMC Pulm. Med. 2017, 17, 70. [CrossRef]

15. Briard, B.; Bomme, P.; Lechner, B.E.; Mislin, G.L.A.; Lair, V.; Prévost, M.-C.; Latgé, J.-P.; Haas, H.; Beauvais, A. Pseudomonas aeruginosa manipulates redox and iron homeostasis of its microbiota partner Aspergillus fumigatus via phenazines. Sci. Rep. 2015, 5, 8220. [CrossRef]

16. Briard, B.; Rasoldier, V.; Bomme, P.; Elaouad, N.; Guerreiro, C.; Chassagne, P.; Muszkieta, L.; Latgé, J.-P.; Mulard, L.; Beauvais, A. Dirhamnolipids secreted from Pseudomonas aeruginosa modify antifungal susceptibility of Aspergillus fumigatus by inhibiting $\beta 1,3$ glucan synthase activity. ISME J. 2017, 11, 1578-1591. [CrossRef]

17. Nazik, H.; Choudhary, V.; Stevens, D.A. Verapamil Inhibits Aspergillus Biofilm, but Antagonizes Voriconazole. J. Fungi (Basel) 2017, 3, 50. [CrossRef] [PubMed]

18. Elliot, R.P. Some properties of pyoverdine, the water-soluble fluorescent pigment of the pseudomonads. Appl. Microbiol. 1958, 6, 241-246. [CrossRef]

19. Chatterjee, P.; Sass, G.; Swietnicki, W.; Stevens, D.A. Review of Potential Pseudomonas Weaponry, Relevant to the PseudomonasAspergillus Interplay, for the Mycology Community. J. Fungi (Basel) 2020, 6, 81. [CrossRef]

20. Stites, S.W.; Walters, B.; O’Brien-Ladner, A.R.; Bailey, K.; Wesselius, L.J. Increased Iron and Ferritin Content of Sputum from Patients with Cystic Fibrosis or Chronic Bronchitis. Chest 1998, 114, 814-819. [CrossRef]

21. Wang, J.; Lory, S.; Ramphal, R.; Jin, S. Isolation and characterization of Pseudomonas aeruginosa genes inducible by respiratory mucus derived from cystic fibrosis patients. Mol. Microbiol. 1996, 22, 1005-1012. [CrossRef] [PubMed]

22. Nazik, H.; Sass, G.; Ansari, S.R.; Ertekin, R.; Haas, H.; Déziel, E.; Stevens, D.A. Novel intermicrobial molecular interaction: Pseudomonas aeruginosa Quinolone Signal (PQS) modulates Aspergillus fumigatus response to iron. Microbiology 2020, 166, 44-55. [CrossRef]

23. Sass, G.; Ansari, S.R.; Dietl, A.-M.; Déziel, E.; Haas, H.; Stevens, D.A. Intermicrobial interaction: Aspergillus fumigatus siderophores protect against competition by Pseudomonas aeruginosa. PLoS ONE 2019, 14, e0216085. [CrossRef]

24. Bargon, J.; Dauletbaev, N.; Köhler, B.; Wolf, M.; Posselt, H.-G.; Wagner, T.O.F. Prophylactic antibiotic therapy is associated with an increased prevalence of Aspergillus colonization in adult cystic fibrosis patients. Respir. Med. 1999, 93, 835-838. [CrossRef]

25. Denning, D.W.; Park, S.; Lass-Florl, C.; Fraczek, M.G.; Kirwan, M.; Gore, R.; Smith, J.; Bueid, A.; Moore, C.B.; Bowyer, P.; et al. High-frequency Triazole Resistance Found In Nonculturable Aspergillus fumigatus from Lungs of Patients with Chronic Fungal Disease. Clin. Infect. Dis. 2011, 52, 1123-1129. [CrossRef] [PubMed]

26. Morio, F.; Aubin, G.G.; Danner-Boucher, I.; Haloun, A.; Sacchetto, E.; Garcia-Hermoso, D.; Bretagne, S.; Miegeville, M.; Le Pape, P. High prevalence of triazole resistance in Aspergillus fumigatus, especially mediated by TR/L98H, in a French cohort of patients with cystic fibrosis. J. Antimicrob. Chemother. 2012, 67, 1870-1873. [CrossRef]

27. Mortensen, K.L.; Jensen, R.H.; Johansen, H.K.; Skov, M.; Pressler, T.; Howard, S.J.; Leatherbarrow, H.; Mellado, E.; Arendrup, M.C. Aspergillus Species and Other Molds in Respiratory Samples from Patients with Cystic Fibrosis: A Laboratory-Based Study with Focus on Aspergillus fumigatus Azole Resistance. J. Clin. Microbiol. 2011, 49, 2243-2251. [CrossRef] [PubMed]

28. Burgel, P.-R.; Baixench, M.-T.; Amsellem, M.; Audureau, E.; Chapron, J.; Kanaan, R.; Honoré, I.; Dupouy-Camet, J.; Dusser, D.; Klaassen, C.H.; et al. High Prevalence of Azole-Resistant Aspergillus fumigatus in Adults with Cystic Fibrosis Exposed to Itraconazole. Antimicrob. Agents Chemother. 2012, 56, 869-874. [CrossRef]

29. Stevens, D.A.; Moss, R.B.; Hernandez, C.; Clemons, K.V.; Martinez, M. Effect of Media Modified To Mimic Cystic Fibrosis Sputum on the Susceptibility of Aspergillus fumigatus, and the Frequency of Resistance at One Center. Antimicrob. Agents Chemother. 2016, 60, 2180-2184. [CrossRef] [PubMed]

30. Denning, D.W.; Clemons, K.V.; Hanson, L.H.; Stevens, D.A.; Morrison, D.C.; Silverstein, R.; Bright, S.W.; Chen, T.-Y.; Flebbe, L.M.; Lei, M.-G. Restriction Endonuclease Analysis of Total Cellular DNA of Aspergillus fumigatus Isolates of Geographically and Epidemiologically Diverse Origin. J. Infect. Dis. 1990, 162, 1151-1158. [CrossRef] [PubMed]

31. Denning, D.W.; Stevens, D.A. Efficacy of cilofungin alone and in combination with amphotericin B in a murine model of disseminated aspergillosis. Antimicrob. Agents Chemother. 1991, 35, 1329-1333. [CrossRef] 
32. O'Toole, G.A.; Kolter, R. Flagellar and twitching motility are necessary for Pseudomonas aeruginosa biofilm development. Mol. Microbiol. 1998, 30, 295-304. [CrossRef] [PubMed]

33. Lee, D.G.; Urbach, J.M.; Wu, G.; Liberati, N.T.; Feinbaum, R.L.; Miyata, S.; Diggins, L.T.; He, J.; Saucier, M.; Déziel, E.; et al. Genomic analysis reveals that Pseudomonas aeruginosa virulence is combinatorial. Genome Biol. 2006, 7, R90. [CrossRef]

34. Fischer, S.; Klockgether, J.; Losada, P.M.; Chouvarine, P.; Cramer, N.; Davenport, C.F.; Dethlefsen, S.; Dorda, M.; Goesmann, A.; Hilker, R.; et al. Intraclonal genome diversity of the major Pseudomonas aeruginosa clones C and PA14. Environ. Microbiol. Rep. 2016, 8, 227-234. [CrossRef] [PubMed]

35. Stover, C.K.; Pham, X.Q.; Erwin, A.L.; Mizoguchi, S.D.; Warrener, P.; Hickey, M.J.; Brinkman, F.S.L.; Hufnagle, W.O.; Kowalik, D.J.; Lagrou, M.; et al. Complete genome sequence of Pseudomonas aeruginosa PAO1, an opportunistic pathogen. Nature 2000, 406, 959-964. [CrossRef] [PubMed]

36. Ferreira, J.A.G.; Penner, J.C.; Moss, R.B.; Haagensen, J.A.J.; Clemons, K.V.; Spormann, A.M.; Nazik, H.; Cohen, K.; Banaei, N.; Carolino, E.; et al. Inhibition of Aspergillus fumigatus and Its Biofilm by Pseudomonas aeruginosa Is Dependent on the Source, Phenotype and Growth Conditions of the Bacterium. PLoS ONE 2015, 10, e0134692. [CrossRef]

37. Scudiero, D.A.; Shoemaker, R.H.; Paull, K.D.; Monks, A.; Tierney, S.; Nofziger, T.H.; Currens, M.J.; Seniff, D.; Boyd, M.R. Evaluation of a soluble tetrazolium/formazan assay for cell growth and drug sensitivity in culture using human and other tumor cell lines. Cancer Res. 1988, 48, 4827-4833. [PubMed]

38. Eliopoulos, G.M.; Eliopoulos, C.T. Antibiotic combinations: Should they be tested? Clin. Microbiol. Rev. 1988, 1, 139-156. [CrossRef]

39. Zhao, W.; Sachsenmeier, K.; Zhang, L.; Sult, E.; Hollingsworth, R.E.; Yang, H. A New Bliss Independence Model to Analyze Drug Combination Data. J. Biomol. Screen. 2014, 19, 817-821. [CrossRef] [PubMed] 